\title{
Topological indices of carbon graphite and crystal cubic carbon structures via M Polynomials
}

\author{
Wei Gao ${ }^{1}$, Waqas Nazeer ${ }^{2}$, Amna Yousaf ${ }^{3}$ and Shin Min Kang ${ }^{4, *}$
}

1. School of Information Science and Technology, Yunnan Normal University, Kunming 650500, China; gaowei@ynnu.edu.cn

2. Department of Mathematics, Division of Science and Technology, University of Education Lahore Pakistan; nazeer.waqas@ue.edu.pk

3. Department of Mathematics and Statistics, The University of Lahore, Lahore 54000, Pakistan; amnayousaf41@gmail.com

4. Department of Mathematics and RINS, Gyeongsang National University, Jinju 52828, Korea, smkang@gnu.ac.kr

Corresponding Author: nazeer.waqas@ue.edu.pk ; +92-321-4707379

\begin{abstract}
Graph theory plays a crucial role in modeling and designing of chemical structure or chemical network. Chemical Graph theory helps to understand the molecular structure of molecular graph. The molecular graph consists of atoms as vertices and bonds as edges. Topological indices capture symmetry of molecular structures and give it a mathematical language to predict properties such as boiling points, viscosity, the radius of gyrations etc. In this article, we study the chemical graph of carbon Crystal structure of graphite and cubic carbon and compute several degreebased topological indices. Firstly we compute M-Polynomials of these structures and then from these M-polynomials we recover nine degree-based topological indices.
\end{abstract}

Keywords: carbon graphite, crystal cubic carbon, M-polynomial, Zagreb index, Randic index

\section{Introduction}

Graph theory helps in for designing chemical structures and modeling and complex networks. Chemical graph theory helps the study of molecular structure. This theory has played an important role in the field of chemical science. Chemical structures can be represented by graph, where the vertices represent atoms and the edges represent molecular bonds. The Topology Index is a number that represents some useful information about the molecular structure. It is a numerical invariant of the molecular graph and is useful in relation to their biological and physicochemical properties. A wide variety of topological indices was studied and used for theoretical chemistry and pharmaceuticals Researchers. Researchers found that topological index is a powerful and useful tool in the description of molecular structure [1]. 
The researchers focused on the applications of topological indices on different molecular compounds [2-6]. Due to this reason we are motived to study topological indices. Algebraic polynomials have also useful applications in chemistry such as Hosoya polynomial (also called Wiener polynomial) [7] which play a vital role in determining distance-based topological indices. Among other algebraic polynomials, M-polynomial [8] introduced in 2015, plays the same role in determining the closed form of many degree-based topological indices [9-13]. The main advantage of Mpolynomial is the wealth of information that it contains about degree-based graph invariants. For more details see [14-16].

In this paper we compute M-polynomials of carbon graphite and crystal cubic carbon structures by using edge partition of molecular graph of carbon graphite and crystal cubic carbon structures. From these M-polynomials we recover many degree based topological indices of understudy molecular compounds. Note that First and second Zagreb indices of these compounds was computed directly in [14].

\section{Basic Definition and Literature Review}

Definition 1. [8] The M-polynomial of $G$ is defined as:

$$
M(G ; x, y)=\sum_{\delta \leq i \leq j \leq \Delta} m_{i j}(G) x^{i} y^{j},
$$

where $\delta=\operatorname{Min}\left\{d_{v}: v \in V(G)\right\}, \Delta=\operatorname{Max}\left\{d_{v}: v \in V(G)\right\}$, and $m_{i j}(G)$ is the edge $v u \in E(G)$ such that $\left\{d_{v}, d_{u}\right\}=\{i, j\}$.

Weiner, in 1947 approximated the boiling point of alkanes as $\alpha W(G)+\beta P_{3}+\gamma$ where $\alpha, \beta$ and $\gamma$ are empirical constants, $W(G)$ is the Weiner index and $P_{3}$ is the number of paths of length 3 in $G$ [17]. Thus Weiner laid the foundation of Topological index which is also known as connectivity index. A lot of chemical experiments require determining the chemical properties of emerging nanotubes and nanomaterials. Chemical-based experiments reveal that out of more than 140 topological indices no single index in strong enough to determine many physico-chemical properties, although, in combination, these topological indices can do this to some extent. The Wiener index is originally the first and most studied topological index, see for details $[18,19]$.

Randic' index, [20] denoted by $R_{-1 / 2}(G)$ and introduced by Milan Randic' in 1975 is also one of the oldest topological indexes. The Randic' index is defined as $R_{-1 / 2}(G)=\sum_{u v E(G)} \frac{1}{\sqrt{d_{u} d_{v}}}$. In 1998, working independently, Bollobas and Erdos [21] and Amic et al. [22] proposed the generalized Randic' index and has been studied 
extensively by both chemist and mathematicians [23] and many mathematical properties of this index have been discussed in [24]. For a detailed survey we refer the book [25]. The general Randic' index is defined as $R_{\alpha}(G)=\sum_{u \in E(G)} \frac{1}{\left(d_{u} d_{v}\right)^{\alpha}}$, and the inverse Randic' index is defined as $R R_{\alpha}(G)=\sum_{u \in E(G)}\left(d_{u} d_{v}\right)^{\alpha}$. Obviously $R_{-1 / 2}(G)$ is the particular case of $R_{\alpha}(G)$ when $\alpha=-\frac{1}{2}$. The Randić index is the also a most popular most often applied and most studied among all other topological indices. Many papers and books such as [26-28] are written on this topological index. Randić himself wrote two reviews on his Randic index [29,30] and there are three more reviews [31-33]. The suitability of the Randić index for drug design was immediately recognized, and eventually, the index was used for this purpose on countless occasions. The physical reason for the success of such a simple graph invariant is still an enigma, although several more-orless plausible explanations were offered.

Gutman and Trinajstic' introduced first Zagreb index and second Zagreb index, which are defined as: $M_{1}(G)=\sum_{u v E(G)}\left(d_{u}+d_{v}\right)$ and $M_{2}(G)=\sum_{u v E(G)}\left(d_{u} \times d_{v}\right)$ respectively. For detail about these indices we refer [34-38] to the readers. Both the first Zagreb index and the second Zagreb index give greater weights to the inner vertices and edges, and smaller weights to outer vertices and edges which oppose intuitive reasoning. Hence, they were amended as follows [39]: for a simple connected graph $G$, the second modified Zagreb index is defined as: ${ }^{m} M_{2}(G)=\sum_{u \in E(G)} \frac{1}{d(u) d(v)}$.

The symmetric division index [SDD] is one of the 148 discrete Adriatic indices is a good predictor of the total surface area for polychlorobiphenyls, see[40]. The Symmetric division index of a connected graph $G$ is defined as $S D D(G)=\sum_{u v E(G)}\left\{\frac{\min \left(d_{u}, d_{v}\right)}{\max \left(d_{u}, d_{v}\right)}+\frac{\max \left(d_{u}, d_{v}\right)}{\min \left(d_{u}, d_{v}\right)}\right\}$.

Another variant of Randic index is the harmonic index defined as: $H(G)=\sum_{v u \in E(G)} \frac{2}{d_{u}+d_{v}}$. As far as we know, this index first appeared in [41]. Favaron et al. [42] considered the relation between the harmonic index and the eigenvalues of graphs.

The inverse sum-index is the descriptor that was selected in [43] as a significant predictor of total surface area of octane isomers and for which the extremal graphs 
obtained have a particularly simple and elegant structure. The inverse sum-index is defined as: $I(G)=\sum_{v u \in E(G)} \frac{d_{u} d_{v}}{d_{u}+d_{v}}$.

The augmented Zagreb index of $G$ proposed by Furtula et al. [44] is defined as $A(G)=\sum_{v u \in E(G)}\left\{\frac{d_{u} d_{v}}{d_{u}+d_{v}-2}\right\}^{3}$. This graph invariant has proven to be a valuable predictive index in the study of the heat of formation in octanes and heptanes (see [44]), whose prediction power is better than atom-bond connectivity index (please refer to [45-47] for its research background). Moreover, the tight upper and lower bounds for the augmented Zagreb index of chemical trees, and the trees with minimal augmented Zagreb index were obtained in [44].

The following table 1 relates some well-known degree-based topological indices with M-polynomial [8].

Table 1 Derivation of some degree-based topological indices from M-polynomial

\begin{tabular}{cc}
\hline Topological Index & Derivation from $M(G ; x, y)$ \\
\hline First Zagreb index & $\left(D_{x}+D_{y}\right)(M(G ; x, y))_{x=y=1}$ \\
Second Zagreb index & $\left(D_{x} D_{y}\right)(M(G ; x, y))_{x=y=1}$ \\
Modified Second Zagreb index & $\left(S_{x} S_{y}\right)(M(G ; x, y))_{x=y=1}$ \\
Randic' index & $\left(D_{x}^{\alpha} D_{y}^{\alpha}\right)(M(G ; x, y))_{x=y=1}$ \\
Inverse Randic' index & $\left(S_{x}^{\alpha} S_{y}^{\alpha}\right)(M(G ; x, y))_{x=y=1}$ \\
Symmetric Division Index & $\left(D_{x} S_{y}+S_{x} D_{y}\right)(M(G ; x, y))_{x=y=1}$ \\
Harmonic Index & $2 S_{x} J(M(G ; x, y))_{x=1}$ \\
Inverse sum Index & $S_{x} J D_{x} D_{y}(M(G ; x, y))_{x=1}$ \\
Augmented Zagreb Index & $S_{x}{ }^{3} Q_{-2} J D_{x}{ }^{3} D_{y}{ }^{3}(M(G ; x, y))_{x=1}$ \\
\hline
\end{tabular}

where

$D_{x}=x \frac{\partial(f(x, y)}{\partial x}, D_{y}=y \frac{\partial(f(x, y)}{\partial y} \mathcal{S}_{x}=\int_{0}^{x} \frac{f(t, y)}{t} d t S_{y}=\int_{0}^{y} \frac{f(x, t)}{t} d t ? \mathcal{J}(f(x y))=f(x, x)$, :

$Q_{\alpha}(f(x, y))=x^{\alpha} f(x, y)$.

\section{Carbon graphite}

Graphite is an allotrope of carbon. The chemical graph of carbon graphite $\mathrm{CG}(m, n)$ consist of hexagon shapes. The structure of carbon graphite consists of hexagons in the form of layers one after the other, and between these layers, there is a weak bond. The molecular graph of carbon graphite $\operatorname{CG}(m, n)$ for $t$ levels is depicted in Fig. 1. 


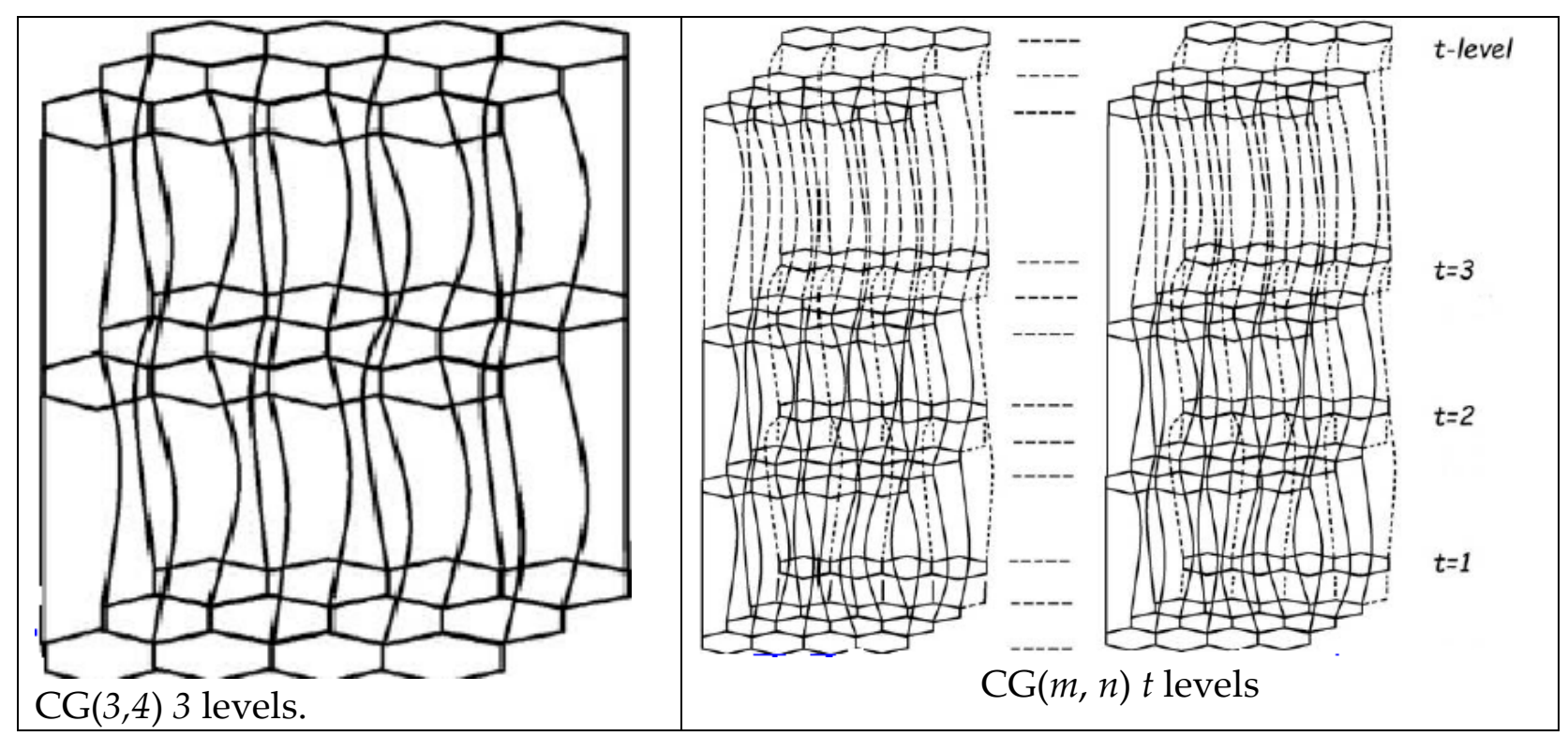

Fig. 1. Carbon graphite graph

Theorem 1. Let $C G(m, n)$ be the carbon graphite. Then

$$
\begin{aligned}
M(G ; x, y)= & 4 x^{2} y^{2}+4(n+t-1) x^{2} y^{3}+4(n t+m-n-t) x^{2} y^{4} \\
& +(4 m+4 t-10) x^{3} y^{3}+(6 m n+6 m t-14 m-4 n-6 t+12) x^{3} y^{4} \\
& +[(4 m n-3 m-2 n+1) t-7 m n+5 m+4 n-2] x^{4} y^{4}
\end{aligned}
$$

Proof. The edge set of $C G(m, n)$ has following six partitions [14],

$$
\begin{gathered}
E_{1}(G)=\left\{u v \in E(G): \quad d_{u}=d_{v}=2\right\} \\
E_{2}(G)=\left\{u v \in E(G): \quad d_{u}=2, d_{v}=3\right\} \\
E_{3}(G)=\left\{u v \in E(G): \quad d_{u}=2, d_{v}=4\right\} \\
E_{4}(G)=\left\{u v \in E(G): \quad d_{u}=d_{v}=3\right\} \\
E_{5}(G)=\left\{u v \in E(G): \quad d_{u}=3, a d_{v}=4\right\} \\
E_{6}(G)=\left\{u v \in E(G): \quad d_{u}=d_{v}=4\right\}
\end{gathered}
$$

Now 


$$
\begin{aligned}
& \left|E_{\{2,2\}}\right|=4 \\
& \quad\left|E_{\{2,3\}}\right|=4(n+t-1) \\
& \quad\left|E_{\{2,4\}}\right|=4(n t+m-n-t) \\
& \left|E_{\{3,3\}}\right|=4 m+4 t-10 \\
& \left|E_{\{3,4\}}\right|=6 m n+6 m t-14 m-4 n-6 t+12 \\
& \left|E_{\{4,4\}}\right|=(4 m n-3 m-2 n+1) t-7 m n+5 m+4 n-2
\end{aligned}
$$

By definition of M-Polynomial

$$
\begin{aligned}
& M(G ; x, y)=\sum_{i \leq j} m_{i j} x^{i} y^{j} \\
& =\left|E_{1}\right| x^{2} y^{2}+\left|E_{2}\right| x^{2} y^{3}+\left|E_{3}\right| x^{2} y^{4}+\left|E_{4}\right| x^{3} y^{3}+\left|E_{5}\right| x^{3} y^{4}+\left|E_{6}\right| x^{4} y^{4} \\
& =4 x^{2} y^{2}+4(n+t-1) x^{2} y^{3}+4(n t+m-n-t) x^{2} y^{4} \\
& +(4 m+4 t-10) x^{3} y^{3}+(6 m n+6 m t-14 m-4 n-6 t+12) x^{3} y^{4} \\
& +[(4 m n-3 m-2 n+1) t-7 m n+5 m+4 n-2] x^{4} y^{4}
\end{aligned}
$$

Some degree-based topologcal indices of Carbon Graphite CG(m,n) graph are given in the following proposition.

Proposition 1. For the Carbon Graphite CG(m,n) graph we have

1. $M_{1}(G)=16+20(n+t-1)+24(n t+m-n-t)$

$$
\begin{aligned}
& +6(4 m+4 t-10)+7(6 m n+6 m t-14 m-4 n-6 t+12) \\
& +8[(4 m n-3 m-2 n+1) t-7 m n+5 m+4 n-2]
\end{aligned}
$$

2. $M_{2}(G)=16+24(n+t-1)+32(n t+m-n-t)$

$$
\begin{aligned}
& +9(4 m+4 t-10)+12(6 m n+6 m t-14 m-4 n-6 t+12) \\
& +16[(4 m n-3 m-2 n+1) t-7 m n+5 m+4 n-2]
\end{aligned}
$$


3. ${ }^{m} M_{2}=1+\frac{2}{3}(n+t-1)+\frac{1}{2}(n t+m-n-t)$

$$
\begin{aligned}
& +\frac{1}{9}(4 m+4 t-10)+\frac{1}{12}(6 m n+6 m t-14 m-4 n-6 t+12) \\
& +\frac{1}{16}[(4 m n-3 m-2 n+1) t-7 m n+5 m+4 n-2]
\end{aligned}
$$

4. $R_{\alpha}(G)=4 \cdot 2^{\alpha} \cdot 2^{\alpha} x^{2} y^{2}+4 \cdot 3^{\alpha} \cdot 2^{\alpha}(n+t-1)+4 \cdot 4^{\alpha} \cdot 2^{\alpha}(n t+m-n-t)$

$$
+3^{\alpha} \cdot 3^{\alpha}(4 m+4 t-10)+4^{\alpha} \cdot 3^{\alpha}(6 m n+6 m t-14 m-4 n-6 t+12)
$$

$$
+4^{\alpha} \cdot 4^{\alpha}[(4 m n-3 m-2 n+1) t-7 m n+5 m+4 n-2]
$$

5. $R R_{\alpha}(G)=\frac{4}{2^{\alpha} .2^{\alpha}}+\frac{4}{3^{\alpha} \cdot 2^{\alpha}}(n+t-1)+\frac{4}{4^{\alpha} .2^{\alpha}}(n t+m-n-t)$

$$
\begin{aligned}
& +\frac{1}{3^{\alpha} \cdot 3^{\alpha}}(4 m+4 t-10)+\frac{1}{4^{\alpha} \cdot 3^{\alpha}}(6 m n+6 m t-14 m-4 n-6 t+12) \\
& +\frac{1}{4^{\alpha} \cdot 4^{\alpha}}[(4 m n-3 m-2 n+1) t-7 m n+5 m+4 n-2]
\end{aligned}
$$

6. $\operatorname{SSD}(G)=8+\frac{26}{3}(n+t-1)+10(n t+m-n-t)$

$$
\begin{aligned}
& +2(4 m+4 t-10)+\frac{25}{12}(6 m n+6 m t-14 m-4 n-6 t+12) \\
& +2[(4 m n-3 m-2 n+1) t-7 m n+5 m+4 n-2]
\end{aligned}
$$

7. $H(G)=2+\frac{8}{5}(n+t-1)+\frac{4}{3}(n t+m-n-t)$

$$
\begin{aligned}
& +\frac{1}{3}(4 m+4 t-10)+\frac{2}{7}(6 m n+6 m t-14 m-4 n-6 t+12) \\
& +\frac{1}{4}[(4 m n-3 m-2 n+1) t-7 m n+5 m+4 n-2]
\end{aligned}
$$

8. $I(G)=4+\frac{24}{5}(n+t-1)+\frac{16}{3}(n t+m-n-t)$

$$
\begin{aligned}
& +\frac{3}{2}(4 m+4 t-10)+\frac{12}{7}(6 m n+6 m t-14 m-4 n-6 t+12) \\
& +2[(4 m n-3 m-2 n+1) t-7 m n+5 m+4 n-2]
\end{aligned}
$$




$$
\text { 9. } \begin{aligned}
A(G)= & 32+32(n+t-1)+32(n t+m-n-t) \\
& +\frac{729}{64}(4 m+4 t-10)+\frac{1728}{125}(6 m n+6 m t-14 m-4 n-6 t+12) \\
& +\frac{4096}{216}[(4 m n-3 m-2 n+1) t-7 m n+5 m+4 n-2]
\end{aligned}
$$

Proof. Let

$$
\begin{aligned}
M(G ; x, y)= & f(x, y)=4 x^{2} y^{2}+4(n+t-1) x^{2} y^{3}+4(n t+m-n-t) x^{2} y^{4} \\
& +(4 m+4 t-10) x^{3} y^{3}+(6 m n+6 m t-14 m-4 n-6 t+12) x^{3} y^{4} \\
& +[(4 m n-3 m-2 n+1) t-7 m n+5 m+4 n-2] x^{4} y^{4}
\end{aligned}
$$

Then

$$
\begin{aligned}
D_{x}=8 x^{2} y^{2}+ & 8(n+t-1) x^{2} y^{3}+8(n t+m-n-t) x^{2} y^{4} \\
+ & 3(4 m+4 t-10) x^{3} y^{3}+3(6 m n+6 m t-14 m-4 n-6 t+12) x^{3} y^{4} \\
+ & 4[(4 m n-3 m-2 n+1) t-7 m n+5 m+4 n-2] x^{4} y^{4} \\
D_{y}=8 x^{2} y^{2}+ & 12(n+t-1) x^{2} y^{3}+16(n t+m-n-t) x^{2} y^{4} \\
+ & 3(4 m+4 t-10) x^{3} y^{3}+4(6 m n+6 m t-14 m-4 n-6 t+12) x^{3} y^{4} \\
+ & 4[(4 m n-3 m-2 n+1) t-7 m n+5 m+4 n-2] x^{4} y^{4}
\end{aligned}
$$

\section{First Zagreb Index}

$$
\begin{aligned}
M_{1}(G)=16+ & 20(n+t-1)+24(n t+m-n-t) \\
+ & 6(4 m+4 t-10)+7(6 m n+6 m t-14 m-4 n-6 t+12) \\
+ & 8[(4 m n-3 m-2 n+1) t-7 m n+5 m+4 n-2]
\end{aligned}
$$

\section{Second Zagreb Index}

$$
\begin{aligned}
D_{x} D_{y}=16 x^{2} y^{2} & +24(n+t-1) x^{2} y^{3}+32(n t+m-n-t) x^{2} y^{4} \\
+ & 9(4 m+4 t-10) x^{3} y^{3}+12(6 m n+6 m t-14 m-4 n-6 t+12) x^{3} y^{4} \\
+ & 16[(4 m n-3 m-2 n+1) t-7 m n+5 m+4 n-2] x^{4} y^{4}
\end{aligned}
$$




$$
\begin{aligned}
M_{2}(G)=16+ & 24(n+t-1)+32(n t+m-n-t) \\
+ & 9(4 m+4 t-10)+12(6 m n+6 m t-14 m-4 n-6 t+12) \\
+ & 16[(4 m n-3 m-2 n+1) t-7 m n+5 m+4 n-2]
\end{aligned}
$$

\section{Modified Second Zagreb Index}

$$
\begin{aligned}
S_{y}=2 x^{2} y^{2}+ & \frac{4}{3}(n+t-1) x^{2} y^{3}+(n t+m-n-t) x^{2} y^{4} \\
& +\frac{1}{3}(4 m+4 t-10) x^{3} y^{3}+\frac{1}{4}(6 m n+6 m t-14 m-4 n-6 t+12) x^{3} y^{4} \\
& +\frac{1}{4}[(4 m n-3 m-2 n+1) t-7 m n+5 m+4 n-2] x^{4} y^{4}
\end{aligned}
$$

$$
\begin{aligned}
S_{x} S_{y}=\frac{2}{2} x^{2} y^{2} & +\frac{4}{3.2}(n+t-1) x^{2} y^{3}+\frac{1}{2}(n t+m-n-t) x^{2} y^{4} \\
+ & \frac{1}{3.3}(4 m+4 t-10) x^{3} y^{3}+\frac{1}{4.3}(6 m n+6 m t-14 m-4 n-6 t+12) x^{3} y^{4} \\
& +\frac{1}{4.4}[(4 m n-3 m-2 n+1) t-7 m n+5 m+4 n-2] x^{4} y^{4}
\end{aligned}
$$

$$
\begin{aligned}
{ }^{m} M_{2}=1+\frac{2}{3}( & n+t-1)+\frac{1}{2}(n t+m-n-t) \\
+ & \frac{1}{9}(4 m+4 t-10)+\frac{1}{12}(6 m n+6 m t-14 m-4 n-6 t+12) \\
& +\frac{1}{16}[(4 m n-3 m-2 n+1) t-7 m n+5 m+4 n-2]
\end{aligned}
$$

\section{Generalized Randic Index}

$$
\begin{aligned}
D_{y}^{2}=4.2 .2 x^{2} y^{2} & +4.3 .3(n+t-1) x^{2} y^{3}+4.4 .4(n t+m-n-t) x^{2} y^{4} \\
+ & 3.3(4 m+4 t-10) x^{3} y^{3}+4.4(6 m n+6 m t-14 m-4 n-6 t+12) x^{3} y^{4} \\
+ & 4.4[(4 m n-3 m-2 n+1) t-7 m n+5 m+4 n-2] x^{4} y^{4}
\end{aligned}
$$




$$
\begin{aligned}
& D_{y}^{3}=4 \cdot 2 \cdot 2.2 x^{2} y^{2}+4 \cdot 3 \cdot 3.3(n+t-1) x^{2} y^{3}+4 \cdot 4 \cdot 4.4(n t+m-n-t) x^{2} y^{4} \\
& +3.3 .3(4 m+4 t-10) x^{3} y^{3}+4.4 .4(6 m n+6 m t-14 m-4 n-6 t+12) x^{3} y^{4} \\
& +4.4 .4[(4 m n-3 m-2 n+1) t-7 m n+5 m+4 n-2] x^{4} y^{4} \\
& \begin{array}{ll}
\vdots & \vdots \\
\vdots & \vdots
\end{array} \\
& D_{y}^{\alpha}=4.2^{\alpha} x^{2} y^{2}+4.3^{\alpha}(n+t-1) x^{2} y^{3}+4.4^{\alpha}(n t+m-n-t) x^{2} y^{4} \\
& +3^{\alpha}(4 m+4 t-10) x^{3} y^{3}+4^{\alpha}(6 m n+6 m t-14 m-4 n-6 t+12) x^{3} y^{4} \\
& +4^{\alpha}[(4 m n-3 m-2 n+1) t-7 m n+5 m+4 n-2] x^{4} y^{4} \\
& D_{x} D_{y}^{\alpha}=4.2^{\alpha} \cdot 2 x^{2} y^{2}+4.3^{\alpha} \cdot 2(n+t-1) x^{2} y^{3}+4.4^{\alpha} \cdot 2(n t+m-n-t) x^{2} y^{4} \\
& +3^{\alpha} .3(4 m+4 t-10) x^{3} y^{3}+4^{\alpha} .3(6 m n+6 m t-14 m-4 n-6 t+12) x^{3} y^{4} \\
& +4^{\alpha} .4[(4 m n-3 m-2 n+1) t-7 m n+5 m+4 n-2] x^{4} y^{4} \\
& D_{x}^{2} D_{y}^{\alpha}=4 \cdot 2^{\alpha} \cdot 2 \cdot 2 x^{2} y^{2}+4 \cdot 3^{\alpha} \cdot 2 \cdot 2(n+t-1) x^{2} y^{3}+4.4^{\alpha} \cdot 2 \cdot 2(n t+m-n-t) x^{2} y^{4} \\
& +3^{\alpha} .3 .3(4 m+4 t-10) x^{3} y^{3}+4^{\alpha} .3 .3(6 m n+6 m t-14 m-4 n-6 t+12) x^{3} y^{4} \\
& +4^{\alpha} .4 .4[(4 m n-3 m-2 n+1) t-7 m n+5 m+4 n-2] x^{4} y^{4} \\
& D_{x}^{3} D_{y}^{\alpha}=4 \cdot 2^{\alpha} \cdot 2 \cdot 2 \cdot 2 x^{2} y^{2}+4 \cdot 3^{\alpha} \cdot 2 \cdot 2 \cdot 2(n+t-1) x^{2} y^{3}+4 \cdot 4^{\alpha} \cdot 2 \cdot 2 \cdot 2(n t+m-n-t) x^{2} y^{4} \\
& +3^{\alpha} \cdot 3.3 .3(4 m+4 t-10) x^{3} y^{3}+4^{\alpha} \cdot 3 \cdot 3 \cdot 3(6 m n+6 m t-14 m-4 n-6 t+12) x^{3} y^{4} \\
& +4^{\alpha} \cdot 4 \cdot 4.4[(4 m n-3 m-2 n+1) t-7 m n+5 m+4 n-2] x^{4} y^{4} \\
& D_{x}^{\alpha} D_{y}^{\alpha}=4.2^{\alpha} \cdot 2^{\alpha} x^{2} y^{2}+4 \cdot 3^{\alpha} \cdot 2^{\alpha}(n+t-1) x^{2} y^{3}+4.4^{\alpha} \cdot 2^{\alpha}(n t+m-n-t) x^{2} y^{4} \\
& +3^{\alpha} \cdot 3^{\alpha}(4 m+4 t-10) x^{3} y^{3}+4^{\alpha} \cdot 3^{\alpha}(6 m n+6 m t-14 m-4 n-6 t+12) x^{3} y^{4} \\
& +4^{\alpha} \cdot 4^{\alpha}[(4 m n-3 m-2 n+1) t-7 m n+5 m+4 n-2] x^{4} y^{4} \\
& R_{\alpha}(G)=4.2^{\alpha} \cdot 2^{\alpha} x^{2} y^{2}+4.3^{\alpha} \cdot 2^{\alpha}(n+t-1)+4.4^{\alpha} \cdot 2^{\alpha}(n t+m-n-t) \\
& +3^{\alpha} \cdot 3^{\alpha}(4 m+4 t-10)+4^{\alpha} \cdot 3^{\alpha}(6 m n+6 m t-14 m-4 n-6 t+12) \\
& +4^{\alpha} .4^{\alpha}[(4 m n-3 m-2 n+1) t-7 m n+5 m+4 n-2]
\end{aligned}
$$

\section{Inverse Randic' Index}




$$
\begin{aligned}
S_{y}=\frac{4}{2} x^{2} y^{2}+ & \frac{4}{3}(n+t-1) x^{2} y^{3}+\frac{4}{4}(n t+m-n-t) x^{2} y^{4} \\
& +\frac{1}{3}(4 m+4 t-10) x^{3} y^{3}+\frac{1}{4}(6 m n+6 m t-14 m-4 n-6 t+12) x^{3} y^{4} \\
& +\frac{1}{4}[(4 m n-3 m-2 n+1) t-7 m n+5 m+4 n-2] x^{4} y^{4}
\end{aligned}
$$$$
S_{y}^{2}=\frac{4}{2.2} x^{2} y^{2}+\frac{4}{3.3}(n+t-1) x^{2} y^{3}+\frac{4}{4.4}(n t+m-n-t) x^{2} y^{4}
$$$$
+\frac{1}{3.3}(4 m+4 t-10) x^{3} y^{3}+\frac{1}{4.4}(6 m n+6 m t-14 m-4 n-6 t+12) x^{3} y^{4}
$$$$
+\frac{1}{4.4}[(4 m n-3 m-2 n+1) t-7 m n+5 m+4 n-2] x^{4} y^{4}
$$$$
S_{y}^{3}=\frac{4}{2 \cdot 2.2} x^{2} y^{2}+\frac{4}{3 \cdot 3 \cdot 3}(n+t-1) x^{2} y^{3}+\frac{4}{4 \cdot 4 \cdot 4}(n t+m-n-t) x^{2} y^{4}
$$$$
+\frac{1}{3.3 .3}(4 m+4 t-10) x^{3} y^{3}+\frac{1}{4.4 .4}(6 m n+6 m t-14 m-4 n-6 t+12) x^{3} y^{4}
$$$$
+\frac{1}{4.4 .4}[(4 m n-3 m-2 n+1) t-7 m n+5 m+4 n-2] x^{4} y^{4}
$$

$$
\begin{aligned}
S_{y}^{\alpha}=\frac{4}{2^{\alpha}} x^{2} y^{2} & +\frac{4}{3^{\alpha}}(n+t-1) x^{2} y^{3}+\frac{4}{4^{\alpha}}(n t+m-n-t) x^{2} y^{4} \\
& +\frac{1}{3^{\alpha}}(4 m+4 t-10) x^{3} y^{3}+\frac{1}{4^{\alpha}}(6 m n+6 m t-14 m-4 n-6 t+12) x^{3} y^{4} \\
& +\frac{1}{4^{\alpha}}[(4 m n-3 m-2 n+1) t-7 m n+5 m+4 n-2] x^{4} y^{4}
\end{aligned}
$$

$$
\begin{aligned}
S_{x} S_{y}^{\alpha}=\frac{4}{2^{\alpha} .2} & x^{2} y^{2}+\frac{4}{3^{\alpha} .2}(n+t-1) x^{2} y^{3}+\frac{4}{4^{\alpha} .2}(n t+m-n-t) x^{2} y^{4} \\
& +\frac{1}{3^{\alpha} \cdot 3}(4 m+4 t-10) x^{3} y^{3}+\frac{1}{4^{\alpha} .3}(6 m n+6 m t-14 m-4 n-6 t+12) x^{3} y^{4} \\
& +\frac{1}{4^{\alpha} .4}[(4 m n-3 m-2 n+1) t-7 m n+5 m+4 n-2] x^{4} y^{4}
\end{aligned}
$$




$$
\begin{aligned}
& S_{x}^{2} S_{y}^{\alpha}=\frac{4}{2^{\alpha} \cdot 2 \cdot 2} x^{2} y^{2}+\frac{4}{3^{\alpha} \cdot 2.2}(n+t-1) x^{2} y^{3}+\frac{4}{4^{\alpha} \cdot 2 \cdot 2}(n t+m-n-t) x^{2} y^{4} \\
& +\frac{1}{3^{\alpha} \cdot 3 \cdot 3}(4 m+4 t-10) x^{3} y^{3}+\frac{1}{4^{\alpha} \cdot 3 \cdot 3}(6 m n+6 m t-14 m-4 n-6 t+12) x^{3} y^{4} \\
& +\frac{1}{4^{\alpha} .4 .4}[(4 m n-3 m-2 n+1) t-7 m n+5 m+4 n-2] x^{4} y^{4} \\
& S_{x}^{3} S_{y}^{\alpha}=\frac{4}{2^{\alpha} \cdot 2 \cdot 2 \cdot 2} x^{2} y^{2}+\frac{4}{3^{\alpha} \cdot 2 \cdot 2 \cdot 2}(n+t-1) x^{2} y^{3}+\frac{4}{4^{\alpha} \cdot 2 \cdot 2 \cdot 2}(n t+m-n-t) x^{2} y^{4} \\
& +\frac{1}{3^{\alpha} \cdot 3 \cdot 3 \cdot 3}(4 m+4 t-10) x^{3} y^{3}+\frac{1}{4^{\alpha} \cdot 3 \cdot 3 \cdot 3}(6 m n+6 m t-14 m-4 n-6 t+12) x^{3} y^{4} \\
& +\frac{1}{4^{\alpha} \cdot 4 \cdot 4 \cdot 4}[(4 m n-3 m-2 n+1) t-7 m n+5 m+4 n-2] x^{4} y^{4} \\
& S_{x}^{\alpha} S_{y}^{\alpha}=\frac{4}{2^{\alpha} \cdot 2^{\alpha}} x^{2} y^{2}+\frac{4}{3^{\alpha} \cdot 2^{\alpha}}(n+t-1) x^{2} y^{3}+\frac{4}{4^{\alpha} \cdot 2^{\alpha}}(n t+m-n-t) x^{2} y^{4} \\
& +\frac{1}{3^{\alpha} \cdot 3^{\alpha}}(4 m+4 t-10) x^{3} y^{3}+\frac{1}{4^{\alpha} \cdot 3^{\alpha}}(6 m n+6 m t-14 m-4 n-6 t+12) x^{3} y^{4} \\
& +\frac{1}{4^{\alpha} .4^{\alpha}}[(4 m n-3 m-2 n+1) t-7 m n+5 m+4 n-2] x^{4} y^{4} \\
& R R_{\alpha}(G)=\left.S_{x}^{\alpha} S_{y}^{\alpha}\right|_{x=y=1} \\
& R R_{\alpha}(G)=\frac{4}{2^{\alpha} \cdot 2^{\alpha}} x^{2} y^{2}+\frac{4}{3^{\alpha} \cdot 2^{\alpha}}(n+t-1) x^{2} y^{3}+\frac{4}{4^{\alpha} \cdot 2^{\alpha}}(n t+m-n-t) x^{2} y^{4} \\
& +\frac{1}{3^{\alpha} \cdot 3^{\alpha}}(4 m+4 t-10) x^{3} y^{3}+\frac{1}{4^{\alpha} \cdot 3^{\alpha}}(6 m n+6 m t-14 m-4 n-6 t+12) x^{3} y^{4} \\
& +\left.\frac{1}{4^{\alpha} .4^{\alpha}}[(4 m n-3 m-2 n+1) t-7 m n+5 m+4 n-2] x^{4} y^{4}\right|_{x=y=1} \\
& R R_{\alpha}(G)=\frac{4}{2^{\alpha} \cdot 2^{\alpha}}+\frac{4}{3^{\alpha} \cdot 2^{\alpha}}(n+t-1)+\frac{4}{4^{\alpha} \cdot 2^{\alpha}}(n t+m-n-t) \\
& +\frac{1}{3^{\alpha} \cdot 3^{\alpha}}(4 m+4 t-10)+\frac{1}{4^{\alpha} \cdot 3^{\alpha}}(6 m n+6 m t-14 m-4 n-6 t+12) \\
& +\frac{1}{4^{\alpha} .4^{\alpha}}[(4 m n-3 m-2 n+1) t-7 m n+5 m+4 n-2]
\end{aligned}
$$

\section{Symmetric Division Index}




$$
\begin{aligned}
D_{x} S_{y}=4 x^{2} y^{2} & +\frac{8}{3}(n+t-1) x^{2} y^{3}+2(n t+m-n-t) x^{2} y^{4} \\
+ & (4 m+4 t-10) x^{3} y^{3}+\frac{3}{4}(6 m n+6 m t-14 m-4 n-6 t+12) x^{3} y^{4} \\
+ & {[(4 m n-3 m-2 n+1) t-7 m n+5 m+4 n-2] x^{4} y^{4} } \\
S_{x} D_{y}=4 x^{2} y^{2} & +6(n+t-1) x^{2} y^{3}+8(n t+m-n-t) x^{2} y^{4} \\
+ & (4 m+4 t-10) x^{3} y^{3}+\frac{4}{3}(6 m n+6 m t-14 m-4 n-6 t+12) x^{3} y^{4} \\
& +[(4 m n-3 m-2 n+1) t-7 m n+5 m+4 n-2] x^{4} y^{4}
\end{aligned}
$$$$
S S D(G)=8+\frac{26}{3}(n+t-1)+10(n t+m-n-t)
$$$$
\begin{aligned}
& +2(4 m+4 t-10)+\frac{25}{12}(6 m n+6 m t-14 m-4 n-6 t+12) \\
& +2[(4 m n-3 m-2 n+1) t-7 m n+5 m+4 n-2]
\end{aligned}
$$

\section{Harmonic Index}

$$
\begin{aligned}
J=4 x^{4}+4( & +t-1) x^{5}+4(n t+m-n-t) x^{6} \\
+ & (4 m+4 t-10) x^{6}+(6 m n+6 m t-14 m-4 n-6 t+12) x^{7} \\
& +[(4 m n-3 m-2 n+1) t-7 m n+5 m+4 n-2] x^{8} \\
S_{x} J=x^{4}+\frac{4}{5}( & n+t-1) x^{5}+\frac{2}{3}(n t+m-n-t) x^{6} \\
& +\frac{1}{6}(4 m+4 t-10) x^{6}+\frac{1}{7}(6 m n+6 m t-14 m-4 n-6 t+12) x^{7} \\
& +\frac{1}{8}[(4 m n-3 m-2 n+1) t-7 m n+5 m+4 n-2] x^{8}
\end{aligned}
$$




$$
\begin{aligned}
H(G)=2+\frac{8}{5}(n+t-1)+\frac{4}{3}(n t+m-n-t) \\
+\frac{1}{3}(4 m+4 t-10)+\frac{2}{7}(6 m n+6 m t-14 m-4 n-6 t+12) \\
+\frac{1}{4}[(4 m n-3 m-2 n+1) t-7 m n+5 m+4 n-2]
\end{aligned}
$$

\section{Inverse Sum Index}

$$
\begin{aligned}
J D_{x} D_{y}=16 x^{4} & +24(n+t-1) x^{5}+32(n t+m-n-t) x^{6} \\
+ & 9(4 m+4 t-10) x^{6}+12(6 m n+6 m t-14 m-4 n-6 t+12) x^{7} \\
+ & 16[(4 m n-3 m-2 n+1) t-7 m n+5 m+4 n-2] x^{8} \\
S_{x} J D_{x} D_{y}=4 & x^{4}+\frac{24}{5}(n+t-1) x^{5}+\frac{16}{3}(n t+m-n-t) x^{6} \\
+ & \frac{3}{2}(4 m+4 t-10) x^{6}+\frac{12}{7}(6 m n+6 m t-14 m-4 n-6 t+12) x^{7} \\
& +2[(4 m n-3 m-2 n+1) t-7 m n+5 m+4 n-2] x^{8} \\
I(G)=4+ & \frac{24}{5}(n+t-1)+\frac{16}{3}(n t+m-n-t) \\
+ & \frac{3}{2}(4 m+4 t-10)+\frac{12}{7}(6 m n+6 m t-14 m-4 n-6 t+12) \\
& +2[(4 m n-3 m-2 n+1) t-7 m n+5 m+4 n-2]
\end{aligned}
$$

\section{Augmented Zagreb Index}

$$
\begin{aligned}
& D_{x}^{3} D_{y}^{3}=4.2^{3} .2^{3} x^{2} y^{2}+4.3^{3} \cdot 2^{3}(n+t-1) x^{2} y^{3}+4.4^{3} \cdot 2^{3}(n t+m-n-t) x^{2} y^{4} \\
&+ 3^{3} \cdot 3^{3}(4 m+4 t-10) x^{3} y^{3}+4^{3} \cdot 3^{3}(6 m n+6 m t-14 m-4 n-6 t+12) x^{3} y^{4} \\
&+ 4^{3} \cdot 4^{3}[(4 m n-3 m-2 n+1) t-7 m n+5 m+4 n-2] x^{4} y^{4} \\
& J D_{x}^{3} D_{y}^{3}=256 x^{4}+864(n+t-1) x^{5}+2048(n t+m-n-t) x^{6} \\
&+ 729(4 m+4 t-10) x^{6}+1728(6 m n+6 m t-14 m-4 n-6 t+12) x^{7} \\
&+4096[(4 m n-3 m-2 n+1) t-7 m n+5 m+4 n-2] x^{8}
\end{aligned}
$$




$$
\begin{aligned}
Q_{-2} J D_{x}^{3} D_{y}^{3}= & 256 x^{4-2}+864(n+t-1) x^{5-2}+2048(n t+m-n-t) x^{6-2} \\
& +729(4 m+4 t-10) x^{6-2}+1728(6 m n+6 m t-14 m-4 n-6 t+12) x^{7-2} \\
& +4096[(4 m n-3 m-2 n+1) t-7 m n+5 m+4 n-2] x^{8-2} \\
Q_{-2} J D_{x}^{3} D_{y}^{3}= & 256 x^{2}+864(n+t-1) x^{3}+2048(n t+m-n-t) x^{4} \\
& +729(4 m+4 t-10) x^{4}+1728(6 m n+6 m t-14 m-4 n-6 t+12) x^{5} \\
& +4096[(4 m n-3 m-2 n+1) t-7 m n+5 m+4 n-2] x^{6} \\
S_{x} Q_{-2} J D_{x}^{3} D_{y}^{3}= & \frac{256}{2} x^{2}+\frac{864}{3}(n+t-1) x^{3}+\frac{2048}{4}(n t+m-n-t) x^{4} \\
+ & \frac{729}{4}(4 m+4 t-10) x^{4}+\frac{1728}{5}(6 m n+6 m t-14 m-4 n-6 t+12) x^{5} \\
& +\frac{4096}{6}[(4 m n-3 m-2 n+1) t-7 m n+5 m+4 n-2] x^{6} \\
= & \frac{256}{2(2)} x^{2}+\frac{864}{3(3)}(n+t-1) x^{3}+\frac{2048}{4(4)}(n t+m-n-t) x^{4} \\
& +\frac{729}{4(4)}(4 m+4 t-10) x^{4}+\frac{1728}{5(5)}(6 m n+6 m t-14 m-4 n-6 t+12) x^{5} \\
& +\frac{4096}{6(6)}[(4 m n-3 m-2 n+1) t-7 m n+5 m+4 n-2] x^{6}
\end{aligned}
$$

$$
\begin{aligned}
S_{x}^{2} Q_{-2} J D_{x}^{3} D_{y}^{3}= & \frac{256}{4} x^{2}+\frac{864}{9}(n+t-1) x^{3}+\frac{2048}{16}(n t+m-n-t) x^{4} \\
& +\frac{729}{16}(4 m+4 t-10) x^{4}+\frac{1728}{25}(6 m n+6 m t-14 m-4 n-6 t+12) x^{5} \\
& +\frac{4096}{36}[(4 m n-3 m-2 n+1) t-7 m n+5 m+4 n-2] x^{6}
\end{aligned}
$$

$$
\begin{aligned}
S_{x}^{3} Q_{-2} J D_{x}^{3} D_{y}^{3}= & \frac{256}{4(2)} x^{2}+\frac{864}{9(3)}(n+t-1) x^{3}+\frac{2048}{16(4)}(n t+m-n-t) x^{4} \\
& +\frac{729}{16(4)}(4 m+4 t-10) x^{4}+\frac{1728}{25(5)}(6 m n+6 m t-14 m-4 n-6 t+12) x^{5} \\
& +\frac{4096}{36(6)}[(4 m n-3 m-2 n+1) t-7 m n+5 m+4 n-2] x^{6}
\end{aligned}
$$




$$
\begin{aligned}
S_{x}^{3} Q_{-2} J D_{x}^{3} D_{y}^{3}= & \frac{256}{8} x^{2}+\frac{864}{27}(n+t-1) x^{3}+\frac{2048}{64}(n t+m-n-t) x^{4} \\
& +\frac{729}{64}(4 m+4 t-10) x^{4}+\frac{1728}{125}(6 m n+6 m t-14 m-4 n-6 t+12) x^{5} \\
& +\frac{4096}{216}[(4 m n-3 m-2 n+1) t-7 m n+5 m+4 n-2] x^{6}
\end{aligned}
$$

$$
\begin{aligned}
A(G)= & 32 x^{2}+32(n+t-1) x^{3}+32(n t+m-n-t) x^{4} \\
+ & \frac{729}{64}(4 m+4 t-10) x^{4}+\frac{1728}{125}(6 m n+6 m t-14 m-4 n-6 t+12) x^{5} \\
& +\left.\frac{4096}{216}[(4 m n-3 m-2 n+1) t-7 m n+5 m+4 n-2] x^{6}\right|_{x=1}
\end{aligned}
$$

$$
\begin{aligned}
A(G)= & 32+32(n+t-1)+32(n t+m-n-t) \\
+ & \frac{729}{64}(4 m+4 t-10)+\frac{1728}{125}(6 m n+6 m t-14 m-4 n-6 t+12) \\
& +\frac{4096}{216}[(4 m n-3 m-2 n+1) t-7 m n+5 m+4 n-2]
\end{aligned}
$$

\section{Crystal structure cubic carbon}

The structure of crystal cubic carbon consist of cubes. The molecular graph of crystal cubic carbon $\operatorname{CCC}(n)$ for the first level is depicted in Fig. 2. For the second level, the cube is constructed at every end vertex of the first level. The second level of CCC $(n)$ is depicted in Fig. 2. Similarly, this procedure is repeated to get the next level and so on.

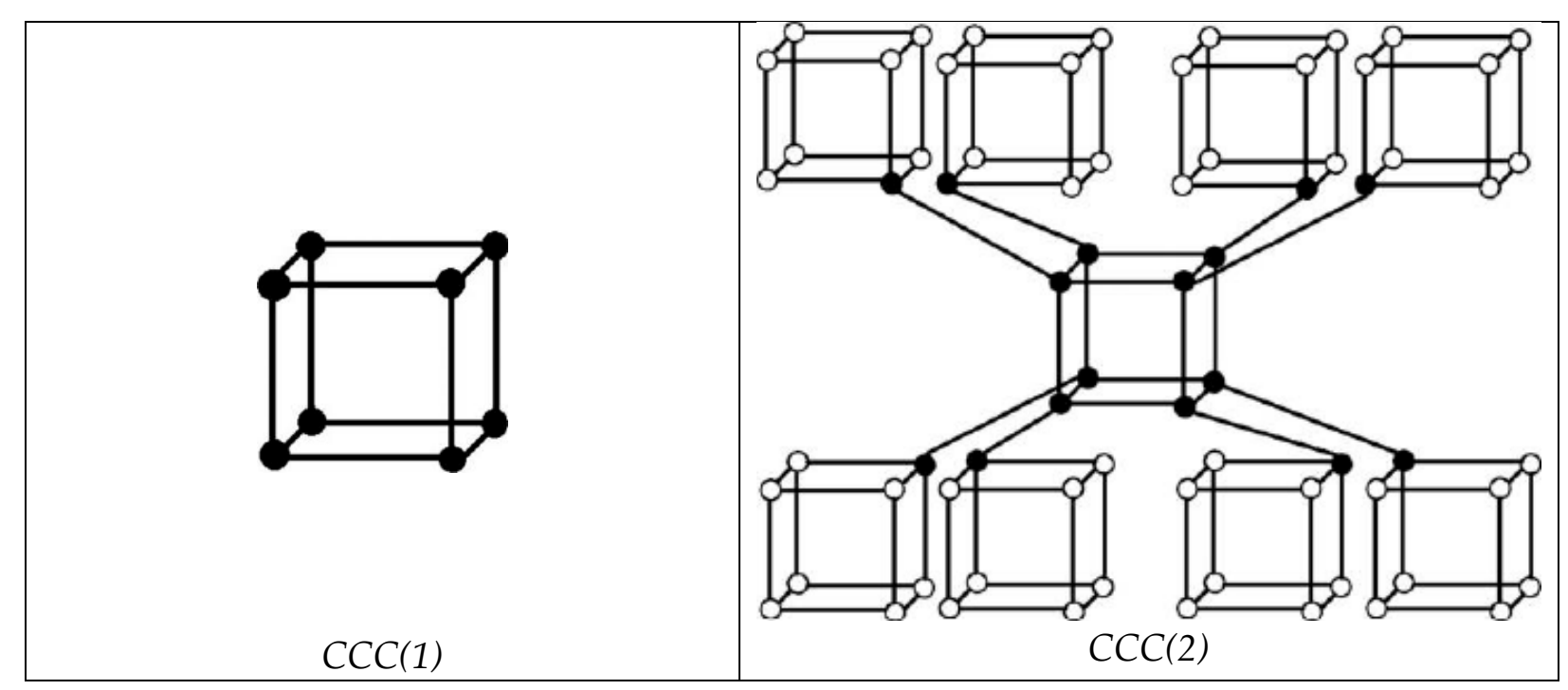


Fig. 2. Crystal structure cubic carbon

Theorem 1. . Let $G$ be the Crystal Cubic Carbon $C C C(n)$ graph.

Then

$M(G ; x, y)=72\left(2^{3}-1\right) n^{-2} x^{3} y^{3}+24\left(2^{3}-1\right) n^{-2} x^{3} y^{4}+\left[12\left(1+\sum_{r=3}^{n} 2^{3}\left(2^{3}-1\right)^{r-3}\right)+8 \sum_{r=0}^{n-2}\left(2^{3}-1\right)^{r}\right] x^{4} y^{4}$

Proof. Let $G=C C C(n)$ be the crystal Cubic Carbon.

The edge set of $C C C(n)$ has following three partitions [14],

$$
\begin{gathered}
E_{1}(G)=\left\{u v \in E(G): \quad d_{u}=d_{v}=3\right\} \\
E_{2}(G)=\left\{u v \in E(G): \quad d_{u}=3, d_{v}=4\right\} \\
E_{3}(G)=\left\{u v \in E(G): \quad d_{u}=d_{v}=4\right\}
\end{gathered}
$$

Now

$$
\begin{gathered}
\left|E_{\{3,3\}}\right|=72\left(2^{3}-1\right) n^{-2} \\
\left|E_{\{3,4\}}\right|=24\left(2^{3}-1\right) n^{-2} \\
\left|E_{\{4,4\}}\right|=12\left[1+\sum_{r=3}^{n} 2^{3}\left(2^{3}-1\right)^{r-3}\right]+8 \sum_{r=0}^{n-2}\left(2^{3}-1\right)^{r}
\end{gathered}
$$

Now by definition of M-polynomial, we have

$$
\begin{aligned}
& M(G ; x, y)=\sum_{i \leq j} m_{i j} x^{i} y^{j} \\
& =\left|E_{1}\right| x^{3} y^{3}+\left|E_{2}\right| x^{3} y^{4}+\left|E_{3}\right| x^{4} y^{4} \\
& =72\left(2^{3}-1\right) n^{-2} x^{3} y^{3}+24\left(2^{3}-1\right) n^{-2} x^{3} y^{4}+\left[12\left(1+\sum_{r=3}^{n} 2^{3}\left(2^{3}-1\right)^{r-3}\right)+8 \sum_{r=0}^{n-2}\left(2^{3}-1\right)^{r}\right] x^{4} y^{4}
\end{aligned}
$$

Now we compute some degree-based topologcal indices of the Crystal Cubic Carbon graph from this M-polynomial

Proposition 2. For the $\mathrm{G}=$ Crystal Cubic Carbon CCC(n) graph. We Have 
1. $M_{1}(G)=600\left(2^{3}-1\right) n^{-2}+8\left[12\left(1+\sum_{r=3}^{n} 2^{3}\left(2^{3}-1\right)^{r-3}\right)+8 \sum_{r=0}^{n-2}\left(2^{3}-1\right)^{r}\right]$

2. $M_{2}(G)=936\left(2^{3}-1\right) n^{-2}+16\left[12\left(1+\sum_{r=3}^{n} 2^{3}\left(2^{3}-1\right)^{r-3}\right)+8 \sum_{r=0}^{n-2}\left(2^{3}-1\right)^{r}\right]$

3. ${ }^{m} M_{2}=10\left(2^{3}-1\right) n^{-2}+\frac{1}{16}\left[12\left(1+\sum_{r=3}^{n} 2^{3}\left(2^{3}-1\right)^{r-3}\right)+8 \sum_{r=0}^{n-2}\left(2^{3}-1\right)^{r}\right]$

4. $R_{\alpha}(G)=72 \cdot 3^{\alpha} \cdot 3^{\alpha}\left(2^{3}-1\right) n^{-2}+24 \cdot 4^{\alpha} \cdot 3^{\alpha}\left(2^{3}-1\right) n^{-2}+4^{\alpha} \cdot 4^{\alpha}\left[12\left(1+\sum_{r=3}^{n} 2^{3}\left(2^{3}-1\right)^{r-3}\right)+8 \sum_{r=0}^{n-2}\left(2^{3}-1\right)^{r}\right]$

5. $R R_{\alpha}(G)=\frac{72}{3^{2 \alpha}}\left(2^{3}-1\right) n^{-2}+\frac{24}{4^{\alpha} \cdot 3^{\alpha}}\left(2^{3}-1\right) n^{-2}+\frac{1}{4^{2 \alpha}}\left[12\left(1+\sum_{r=3}^{n} 2^{3}\left(2^{3}-1\right)^{r-3}\right)+8 \sum_{r=0}^{n-2}\left(2^{3}-1\right)^{r}\right]$

6. $S S D(G)=194\left(2^{3}-1\right) n^{-2}+2\left[12\left(1+\sum_{r=3}^{n} 2^{3}\left(2^{3}-1\right)^{r-3}\right)+8 \sum_{r=0}^{n-2}\left(2^{3}-1\right)^{r}\right]$

7. $H(G)=\frac{216}{7}\left(2^{3}-1\right) n^{-2}+\frac{1}{4}\left[12\left(1+\sum_{r=3}^{n} 2^{3}\left(2^{3}-1\right)^{r-3}\right)+8 \sum_{r=0}^{n-2}\left(2^{3}-1\right)^{r}\right]$

8. $I(G)=\frac{1044}{7}\left(2^{3}-1\right) n^{-2}+2\left[12\left(1+\sum_{r=3}^{n} 2^{3}\left(2^{3}-1\right)^{r-3}\right)+8 \sum_{r=0}^{n-2}\left(2^{3}-1\right)^{r}\right]$

9. $A(G)=\frac{13122}{16}\left(2^{3}-1\right) n^{-2}+\frac{41472}{125}\left(2^{3}-1\right) n^{-2}+\frac{4096}{216}\left[12\left(1+\sum_{r=3}^{n} 2^{3}\left(2^{3}-1\right)^{r-3}\right)+8 \sum_{r=0}^{n-2}\left(2^{3}-1\right)^{r}\right]$

\section{Conclusions}

We have recovered nine degree based topological indices of Carbon graphite and crystal cubic carbon structures from their M-polynomials. We have used edge partition of these compounds to compute M-polynomials. Our results helps to predict many physical properties of understudy chemical compounds for example Randic index is useful for determining physio-chemical properties of alkanes as noticed by chemist Melan Randic in 1975. He noticed the correlation between the Randic index R and 
several physico-chemical properties of alkanes like, enthalpies of formation, boiling points, chromatographic retention times, vapor pressure and surface areas.

\section{References}

1. Ali, A., Bhatti, A., \& Raza, Z. (2016). The augmented Zagreb index, vertex connectivity and matching number of graphs. Bulletin of the Iranian Mathematical Society, 42(2), 417-425.

2. Ali, A., Bhatti, A. A., \& Raza, Z. (2016). Topological Study of Tree-Like Polyphenylene Systems, Spiro Hexagonal Systems and Polyphenylene Dendrimer Nanostars. Quantum Matter, 5(4), 534-538.

3. Raza, Z. (2016). The Edge Version of Geometric Arithmetic Index of Polyomino Chains of 8-Cycles and Arbitrary Carbon Nanocones. Journal of Computational and Theoretical Nanoscience, 13(11), 8455-8459.

4. Brückler, F. M., Došli' 'c, T., Graovac, A. \& Gutman, I. On a class of distance-based molecular structure descriptors. Chem. Phys. Lett. 503, 336-338 (2011).

5. Deng, H., Yang, J. \& Xia, F. A general modeling of some vertex-degree based topological indices in benzenoid systems and phenylenes. Comp. Math. Appl. 61, 3017-3023 (2011).

6. Zhang, H. \& Zhang, F. The Clar covering polynomial of hexagonal systems. Discret. Appl. Math. 69, 147-167 (1996).

7. Gutman, I. Some properties of the Wiener polynomials. Graph Theory Notes New York. 125, 13-18 (1993).

8. Deutsch, E. \& Klavzar, S. M-Polynomial, and degree-based topological indices. Iran. J. Math. Chem. 6, 93-102 (2015).

9. Munir, M., Nazeer, W., Rafique, S. \& Kang, S. M. M-polynomial and related topological indices of Nanostar dendrimers. Symmetry. 8(9), 97; 10.3390/sym8090097 (2016).

10. Munir, M., Nazeer, W., Rafique, S., Nizami, A. R. \& Kang, S. M. M-polynomial and degree-based topological indices of titania nanotubes. Symmetry. 8(11), 117; 10.3390/sym8110117 (2016).

11. Munir, M., Nazeer, W., Rafique, S. \& Kang, S. M. M-Polynomial and DegreeBased Topological Indices of Polyhex Nanotubes. Symmetry. 8(12), 149; 10.3390/sym8120149 (2016).

12. Ajmal, M.; Nazeer, W.; Munir, M.; Kang, S. M.; Kwun, Y. C. M-polynomials and topological indices of generalized prism and toroidal polyhex networks. Under review, Symmetry.

13. Munir, M., Nazeer, W., Shahzadi, S. \& Kang, S. M. Some invariants of circulant graphs. Symmetry. 8(11), 134; 10.3390/sym8110134 (2016). 
14. Baig, A. Q., Imran, M., Khalid, W., \& Naeem, M. (2017). Molecular description of carbon graphite and crystal cubic carbon structures. Canadian Journal of Chemistry, 95(6), 674-686.

15. S. Alikhani, R. Hasni, N.E. Arif, On the atom-bond connectivity index of some families of dendrimers, J. Comput. Theor. Nanosci.,11 (2014) 18021805.

16. A. R. Ashra_M. Mirzargar, PI, Szeged and edge Szeged indices of an in_nite family of nanostarsdendrimers, Indian J. Chem., 47A (2008) 538541. 13.

17. Wiener, H. Structural determination of paraffin boiling points. J. Am. Chem. Soc. 69, 17-20 (1947).

18. Dobrynin, A. A., Entringer, R. E Gutman, I. Wiener index of trees: theory and applications. Acta Appl. Math. 66, 211-249 (2001).

19. Gutman, I. \& Polansky, O. E. Mathematical Concepts in Organic Chemistry. (Springer-Verlag New York, USA, 1986).

20. Randic, M. On the characterization of molecular branching. J. Am. Chem. Soc. 97, 6609- 6615 (1975).

21. Bollobas, B. \& Erdos, P. Graphs of extremal weights. Ars. Combin. 50, 225-233 (1998).

22. Amic, D., Beslo, D., Lucic, B., Nikolic, S. \& Trinajstic', N. The Vertex-Connectivity Index Revisited. J. Chem. Inf. Comput. Sci. 38, 819-822 (1998).

23. Hu, Y., Li, X., Shi, Y., Xu, T. \& Gutman, I. On molecular graphs with smallest and greatest zeroth-Corder general randic index. MATCH Commun. Math. Comput. Chem. 54, 425-434 (2005).

24. Caporossi, G., Gutman, I., Hansen, P. \& Pavlovic, L. Graphs with maximum connectivity indexComput. Biol. Chem. 27, 85-90 (2003).

25. Li, X. \& Gutman, I. Mathematical Chemistry Monographs, No 1. (Kragujevac, 2006).

26. Kier, L. B. \& Hall, L. H. Molecular Connectivity in Chemistry and Drug Research. (Academic Press, New York, 1976).

27. Kier, L. B. \& Hall, L. H. Molecular Connectivity in Structure-Activity Analysis. (Wiley, New York, 1986).

28. Li, X. \& Gutman, I. Mathematical Aspects of Randic-Type Molecular Structure Descriptors. (Univ. Kragujevac, Kragujevac 2006).

29. Randić, M. On History of the Randić Index and Emerging Hostility toward Chemical Graph Theory. MATCH Commun. Math. Comput. Chem. 59, 5-124 (2008).

30. Randić, M. The Connectivity Index 25 Years After.J.Mol.Graphics Modell. 20, 19-35 (2001).

31. Gutman, I. \& Furtula, B. Recent Results in the Theory of Randić Index. (Univ. Kragujevac, Kragujevac 2008).

32. Li, X. \& Shi, Y. A survey on the Randic index. MATCH Commun. Math. Comput. Chem. 59, 127-156 (2008). 
33. Li, X., Shi, Y. \& Wang, L. in: Recent Results in the Theory of Randić Index, I. Gutman and B. Furtula (Eds.) 9-47 (Univ. Kragujevac, Kragujevac 2008).

34. Nikolić, S., Kovačević, G., Miličević, A. \& Trinajstić, N. The Zagreb indices 30 years after. Croat. Chem. Acta 76, 113-124 (2003).

35. Gutman, I. \& Das, K. C. The first Zagreb indices 30 years after. MATCH Commun. Math. Comput. Chem. 50, 83-92 (2004).

36. Das, K. \& Gutman, I. Some Properties of the Second Zagreb Index. MATCH Commun. Math. Comput. Chem. 52, 103-112 (2004).

37. Trinajstic, N.,Nikolic, S., Milicevic, A. \&Gutman, I. On Zagreb indices. Kem. Ind. 59, 577-589 (2010).

38. Vuki cevi'c, D. \& Graovac, A. Valence connectivities versus Randi'c, Zagreb and modified Zagreb index: A linear algorithm to check discriminative properties of indices in acyclic molecular graphs. Croat. Chem. Acta. 77, 501-508 (2004).

39. A. Milicevic, S. Nikolic, N. Trinajstic, On reformulated Zagreb indices, Mol. Divers. 8 (2004) 393-399.

40. Gupta, C. K.; Lokesha, V.; Shwetha, S. B.; Ranjini, P. S. On the Symmetric Division deg Index of Graph. Southeast Asian Bulletin of Mathematics. 2016, Vol. 40 Issue 1, p59-80. 22p.

41. S., Fajtlowicz. On conjectures of Graffiti-II. Congr. Numer., 60 (1987), pp. 187197

42. O. Favaron, M. Mahéo, J.F. Saclé, Some eigenvalue properties in graphs (conjectures of Graffiti-II). Discrete Math., 111 (1993), pp. 197-220.

43. A. T. Balaban, Highly discriminating distance based numerical descriptor, Chem. Phys. Lett. 89 (1982), 399-404.

44. B. Furtula, A. Graovac, D. Vukicevi'c, Augmented Zagreb index, J. Math. Chem. 48 (2010) 370-380.

45. K. C. Das, Atom-bond connectivity index of graphs, Discr. Appl. Math. 158 (2010) 1181-1188.

46. E. Estrada, L. Torres, L. Rodr'iguez, I. Gutman, An atom-bond connectivity index: Modeling the enthalpy of formation of alkanes, Indian J. Chem. 37A (1998) 849-855.

47. E. Estrada, Atom-bond connectivity and the energetic of branched alkanes, Chem. Phys. Lett. 463 (2008) 422-425.

48. Shirdel, G. H, Pour, H. R, Sayadi, A. M. The hyper-Zagreb index of graph operations, Iran J Math Chem 2013;4(2): 213-20

49. M. Ghorbani, N. Azimi, Note on multiple Zagreb indices, Iran. J. Math. Chem. 3 (2) (2012) 137-143.

50. Li, X.; Shi, Y. A Survey on the Randic Index. MATCH Commun. Math. Comput.

Chem. 2008, 59, 127-156. 